\title{
New efficient synthesis of polysubstituted 3,4-dihydroquinazolines and 4H-3,1-benzothiazines through a Passerini/Staudinger/aza-Wittig/addition/nucleophilic substitution sequence
}

\author{
Long Zhao, Mao-Lin Yang, Min Liu and Ming-Wu Ding ${ }^{*} \S$
}

\author{
Full Research Paper \\ Address: \\ Key Laboratory of Pesticide \& Chemical Biology of Ministry of \\ Education, Hubei International Scientific and Technological \\ Cooperation Base of Pesticide and Green Synthesis, Central China \\ Normal University, Wuhan, 430079, P. R. China \\ Email: \\ Ming-Wu Ding ${ }^{*}$ - mwding@mail.ccnu.edu.cn \\ * Corresponding author \\ § Fax: +86 (27) 67862041 \\ Keywords: \\ aza-Wittig reaction; 3,4-dihydroquinazoline; 4H-3,1-benzothiazine; \\ nucleophilic substitution; Passerini reaction; Staudinger reaction \\ Beilstein J. Org. Chem. 2022, 18, 286-292. \\ https://doi.org/10.3762/bjoc.18.32 \\ Received: 05 January 2022 \\ Accepted: 24 February 2022 \\ Published: 04 March 2022 \\ Associate Editor: D. Spring \\ (C) 2022 Zhao et al.; licensee Beilstein-Institut. \\ License and terms: see end of document.
}

\begin{abstract}
A new efficient synthesis of polysubstituted 3,4-dihydroquinazolines and 4H-3,1-benzothiazines via sequential Passerini/ Staudinger/aza-Wittig/addition/nucleophilic substitution reaction has been developed. The three-component Passerini reactions of 2-azidobenzaldehydes $\mathbf{1}$, benzoic acid (2), and isocyanides $\mathbf{3}$ produced the azide intermediates $\mathbf{4}$, which were treated sequentially with triphenylphosphine, isocyanates (or $\mathrm{CS}_{2}$ ), and secondary amines to give polysubstituted 3,4-dihydroquinazolines $\mathbf{8}$ and 4H-3,1-benzothiazines 11 in good overall yields through consecutive Passerini/Staudinger/aza-Wittig/addition/nucleophilic substitution reactions.
\end{abstract}

\section{Introduction}

The chemistry of 3,4-dihydroquinazolines and 4H-3,1-benzothiazines is of constant interest owing to the occurrence of these ring systems in various biologically important compounds (Figure 1). A number of 3,4-dihydroquinazolines were found to show remarkable anticancer [1], antiviral [2], antidepressant [3], antifungal [4], selective somatostatin 2 (ss2) agonistical [5], $\beta$-site amyloid precursor protein cleaving enzyme 1 (BACE-1) inhibitive [6], and cholinesterase enzyme inhibitive activities [7]. The 3,4-dihydroquinazoline skeleton also exists in some natural products such as vasicine and vasicoline [8]. Some 4H-3,1-benzothiazine derivatives have also received attention due to their good biological activities, including anticancer [9], 
<smiles>O=C(CNCc1ccc(F)cc1)CC1c2ccccc2N=C(N2CCN(CCN3CCCC3)CC2)N1c1ccc(C2CCCCC2)cc1</smiles>

anticancer<smiles>Oc1ccc(C2=Nc3ccc(Cl)cc3C(O)(c3ccccc3)S2)c(O)c1</smiles>

antifungal

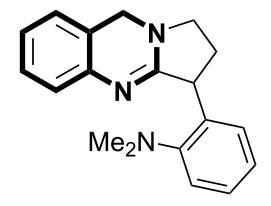

vasicoline

Figure 1: Some bioactive 3,4-dihydroquinazolines and $4 \mathrm{H}-3,1$ benzothiazines.

neuroprotective [10], antiproliferative and antifungal activities [11]. Due to the significant bioactive properties of the 3,4-dihydroquinazoline and 4H-3,1-benzothiazine moieties, many preparation procedures have appeared in the literature for the synthesis of their derivatives [12-22]. For example (Scheme 1), a one-pot $\mathrm{Tf}_{2} \mathrm{O}$-mediated assembly of amides, amines, and ketones provided 3,4-dihydroquinazolines in good yields via successive triflic anhydride-mediated amide dehydration, ketimine addition, and Pictet-Spengler-like cyclization processes [12]. Some 4-substituted 3,4-dihydroquinazolines were prepared by copper-catalyzed oxidative cross coupling of hydroxy intermediates with various nucleophiles [13]. Other 3,4-dihydroquinazolines were also obtained efficiently by intramolecular aza-Wittig reactions [14]. Some 4H-3,1-benzothiazines were prepared by intramolecular thia-Michael addition with broad reaction scopes [19]. The rearrangement of 2-isothiocyano triarylmethanes in the presence of $\mathrm{AlCl}_{3}$ were also used for the synthesis 2,4-diaryl-4H-3,1-benzothiazines through aromatic ring transfer [20]. A facile protocol towards the synthesis of $4 H-3,1$-benzothiazines was established by using

a)

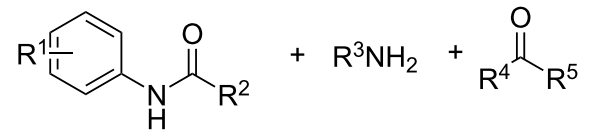

1. $4 \AA$ mol sieves

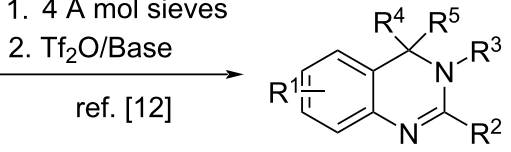

b)

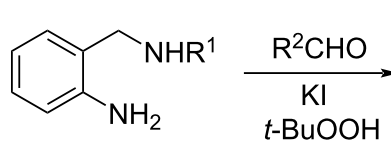<smiles>[R]C1=Nc2ccccc2C(O)N1[R]</smiles><smiles>[R]C1=Nc2ccccc2C(N)N1[R]</smiles>

c)

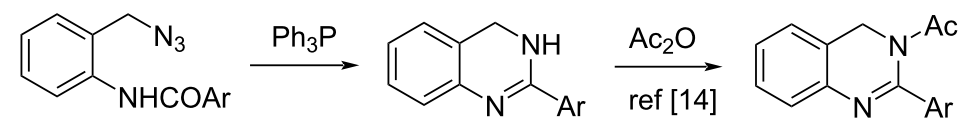

d)

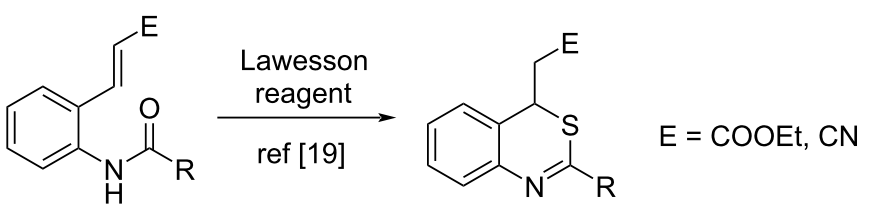

e)

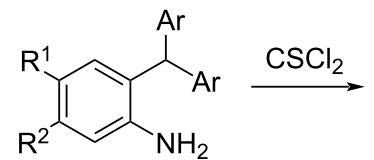<smiles>[R]c1cc(N=S)c(C(Br)[Al][Na])cc1[R]</smiles><smiles>[R]c1cc2c(cc1[R])C([Al])SC([Al])=N2</smiles>

f)<smiles>[R16]S(=O)(=O)c1ccccc1CO</smiles><smiles>[R]NC(=S)Nc1ccccc1CO</smiles>

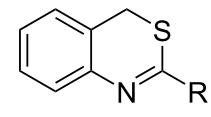


a $\mathrm{P}\left(\mathrm{NMe}_{2}\right)_{3}$-mediated $\mathrm{C}-\mathrm{N} / \mathrm{C}-\mathrm{S}$ bond formation reaction of 2-aminobenzyl alcohol with isothiocyanates under aerobic conditions [21]. Despite of the above achievements, the development of new efficient methods for the synthesis of polysubstituted 3,4-dihydroquinazolines and 4H-3,1-benzothiazines under mild reaction conditions is still of high demand in the discovery of biologically active compounds.

The Passerini reaction is an isocyanide-based multicomponent reaction, which has been used in preparing various $\alpha$-acyloxy adducts starting from aldehydes, a carboxylic acid, and a isonitrile as the three components [23]. The sequences of Passerini reactions, followed by post-condensation reactions, constitute useful synthetic methods in the preparation of structurally diverse heterocyclic compounds [24-29]. The aza-Wittig reaction has also been utilized widely in preparation of various heterocycles under mild neutral conditions [30-32]. Recently we have reported the synthesis of $3 H$-2-benzoxepin-1-ones, $4 H-3,1$-benzoxazines and oxazoles by combination of a Passerini with an intramolecular aza-Wittig reaction [33-35]. Continuing our interest in the synthesis of $N$-heterocycles via the aza-Wittig reaction and multicomponent reactions [36-38], we wish to report herein a facile synthesis of polysubstituted 3,4-dihydroquinazolines and 4H-3,1-benzothiazines via sequential Passerini/Staudinger/aza-Wittig/addition/nucleophilic substitution reactions. Compared with the synthetic method to 4H-3,1-benzothiazines in Scheme 1f, we provide another new sequential synthetic route to $4 H-3,1$-benzothiazines, especially for $N, N$-disubstituted 2-amino-4H-3,1-benzothiazines.

\section{Results and Discussion}

We initially selected 2-azidobenzaldehyde (1a), benzoic acid (2a) and tert-butyl isocyanide (3a) as the reactants (Scheme 2). When a mixture of $\mathbf{1 a}, \mathbf{2 a}$, and $\mathbf{3 a}$ in $\mathrm{CH}_{2} \mathrm{Cl}_{2}$ was stirred at room temperature for $48 \mathrm{~h}$, the three-component Passerini reaction was carried out smoothly and the azide $4 \mathbf{a}(\mathrm{R}=\mathrm{Ph})$ was finally obtained in $87 \%$ yield. Compound $4 \mathbf{a}$ was then allowed to react with triphenylphosphine in $\mathrm{CH}_{2} \mathrm{Cl}_{2}$ at room temperature for $2 \mathrm{~h}$ to produce the iminophosphorane $\mathbf{5 a}$ by Staudinger reaction. Aza-Wittig reaction of 5a with phenyl isocyanate generated carbodiimide $\mathbf{6 a}$, which was then treated with diethylamine to form the guanidine intermediate 7a. In the presence of $\mathrm{K}_{2} \mathrm{CO}_{3}$ in $\mathrm{CH}_{3} \mathrm{CN}$ at refluxing temperature, the 3,4-dihydroquinazoline 8a was finally obtained in $84 \%$ yield (Table 1, entry 1 , the overall yield is $73 \%$ ) by intramolecular nucleophilic substitution. The reaction conditions for the transformation of guanidine intermediate 7a into 3,4-dihydroquinazoline 8a was then optimized (Table 1). As $\mathrm{K}_{2} \mathrm{CO}_{3}$ in different solvents (DMF, $\mathrm{CH}_{2} \mathrm{Cl}_{2}$ and toluene) were used, $0-72 \%$ yields of the product 8a were obtained (Table 1, entries 2-4). Utilizing a stronger base $(\mathrm{NaOH}$ and EtONa) resulted in a dark solution and no product was received (entries 5 and 6 ) owning to side reactions under the stronger base conditions. No product $8 \mathbf{a}$ was obtained when $\mathrm{NEt}_{3}$ in $\mathrm{CH}_{3} \mathrm{CN}$ was used (Table 1, entry 7) probably due to the weaker basic conditions. The effect of different $\mathrm{R}$ groups on the reaction yield was also investigated. With $\mathrm{R}=$ methyl, no product $\mathbf{8 a}$ was obtained in the presence of $\mathrm{K}_{2} \mathrm{CO}_{3} / \mathrm{CH}_{3} \mathrm{CN}$ probably due to the lower reactivity of the -OAc leaving group. In case when $\mathrm{R}$ was a $4-\mathrm{NO}_{2} \mathrm{C}_{6} \mathrm{H}_{4}$ group, $86 \%$ yield of the product $8 \mathbf{a}$ was obtained, however, in this case the Passerini product $4 \mathbf{a}\left(\mathrm{R}=4-\mathrm{NO}_{2} \mathrm{C}_{6} \mathrm{H}_{4}\right)$ was obtained only in $62 \%$ yield and the overall yield of product $8 \mathbf{a}$ was $53 \%$. There-

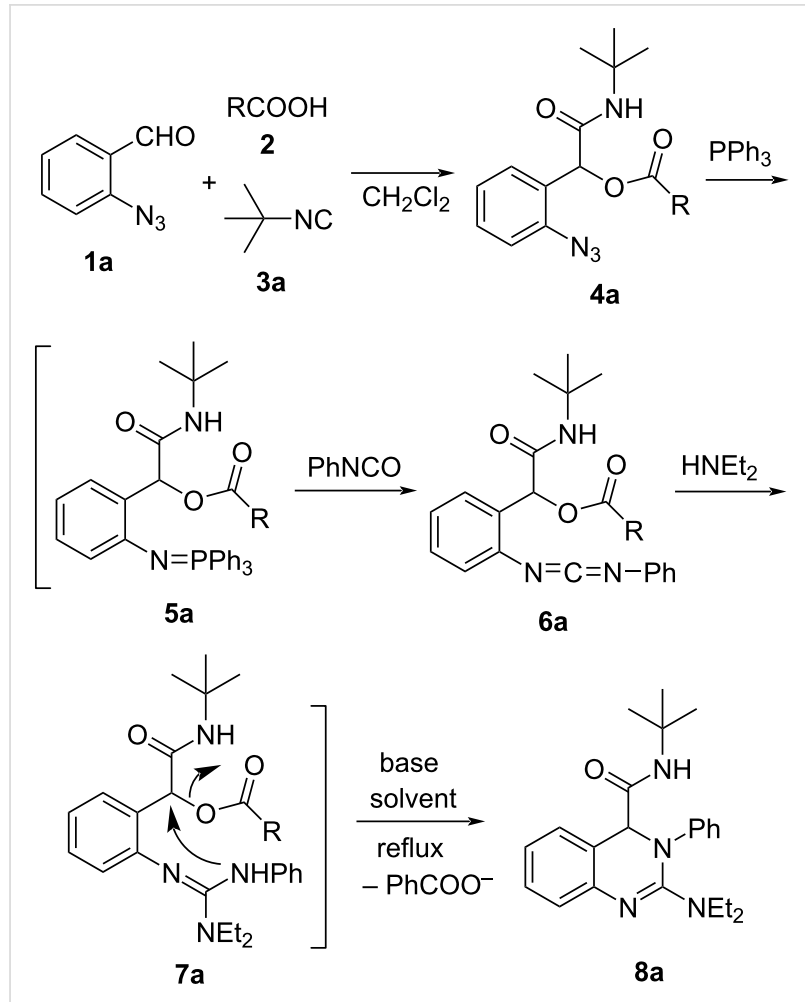

Scheme 2: Preparation of 3,4-dihydroquinazoline $\mathbf{8 a}$

\begin{tabular}{|c|c|c|c|}
\hline entry & $\mathrm{R}$ & Conditions & Yield (\%) \\
\hline 1 & $\mathrm{Ph}$ & $\mathrm{K}_{2} \mathrm{CO}_{3} / \mathrm{CH}_{3} \mathrm{CN}$ & 84 \\
\hline 2 & $\mathrm{Ph}$ & $\mathrm{K}_{2} \mathrm{CO}_{3} / \mathrm{DMF}$ & 72 \\
\hline 3 & $\mathrm{Ph}$ & $\mathrm{K}_{2} \mathrm{CO}_{3} / \mathrm{CH}_{2} \mathrm{Cl}_{2}$ & 0 \\
\hline 4 & $\mathrm{Ph}$ & $\mathrm{K}_{2} \mathrm{CO}_{3} /$ toluene & 41 \\
\hline 5 & $\mathrm{Ph}$ & $\mathrm{NaOH} / \mathrm{CH}_{3} \mathrm{CN}$ & 0 \\
\hline 6 & $\mathrm{Ph}$ & $\mathrm{NaOEt} / \mathrm{EtOH}$ & 0 \\
\hline 7 & $\mathrm{Ph}$ & $\mathrm{NEt}_{3} / \mathrm{CH}_{3} \mathrm{CN}$ & 0 \\
\hline 8 & $\mathrm{Me}$ & $\mathrm{K}_{2} \mathrm{CO}_{3} / \mathrm{CH}_{3} \mathrm{CN}$ & 0 \\
\hline 9 & $4-\mathrm{NO}_{2} \mathrm{C}_{6} \mathrm{H}_{4}$ & $\mathrm{~K}_{2} \mathrm{CO}_{3} / \mathrm{CH}_{3} \mathrm{CN}$ & 86 \\
\hline
\end{tabular}


fore, the reaction conditions of entry 1 in Table 1 were optimal for the above transformation.

The optimal reaction conditions were then utilized for the sequential reactions of different 2 -azidobenzaldehydes $\mathbf{1}$, benzoic acid (2a), isocyanides $\mathbf{3}$, isocyanates and secondary amines. Most of the reactions took place smoothly to give the corresponding 3,4-dihydroquinazolines $\mathbf{8}$ in good yields (Scheme 3 and Table 2). Various isocyanates and secondary amines can be used in the above one-pot cyclization to prepare 3,4-dihydroquinazolines $\mathbf{8}$. As indicated in Table 2, when aromatic isocyanates (Table 2, compounds $\mathbf{8 a - l}, \mathrm{R}^{3}=\mathrm{Ph}$, 4- $\mathrm{ClC}_{6} \mathrm{H}_{4}, 3-\mathrm{MeC}_{6} \mathrm{H}_{4}, 4-\mathrm{MeC}_{6} \mathrm{H}_{4}$ and $4-\mathrm{CF}_{3} \mathrm{OC}_{6} \mathrm{H}_{4}$ ) were used, good yields $(69-86 \%)$ of the products were obtained, whereas moderate yields (54-57\%) were obtained when the more steric secondary amines were utilized (Table 2, compound $\mathbf{8 m}$ and $\left.\mathbf{8 n}, \mathrm{NR}^{4} \mathrm{R}^{5}=\mathrm{N}(\mathrm{Cy})_{2}, \mathrm{~N}(\mathrm{iPr})_{2}\right)$. In cases when aliphatic isocyanates (compounds $\mathbf{8 0 -}-\mathbf{q}, \mathrm{R}^{3}=n$-Bu, cyclohexyl and $\mathrm{PhCH}_{2}$ ) were used, $65-74 \%$ yields of the products were obtained. Even as the steric tert-butyl isocyanate was applied, the 3,4-dihydroquinazoline $8 \mathbf{r}$ was obtained in $42 \%$ yield, but when diphenylamine was used, no product was obtained (compounds 8s, $\mathrm{NR}^{4} \mathrm{R}^{5}=\mathrm{NPh}_{2}$ ).

The aza-Wittig reaction of iminophosphoranes 5 with an excess of $\mathrm{CS}_{2}$ took place smoothly at $40{ }^{\circ} \mathrm{C}$ to produce isothiocyanates

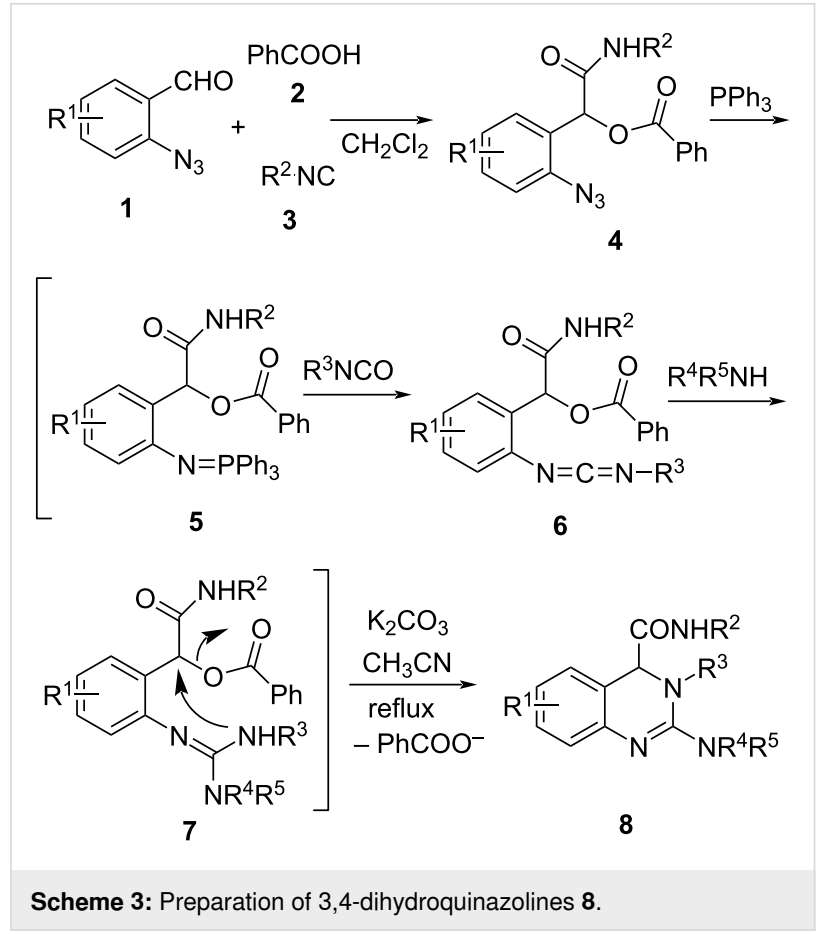

9, which were allowed to react with secondary amines to generate thiourea intermediates 10 . In the presence of $\mathrm{K}_{2} \mathrm{CO}_{3}$ in $\mathrm{CH}_{3} \mathrm{CN}$ at refluxing temperature, thioureas $\mathbf{1 0}$ were also successfully transformed into 4H-3,1-benzothiazines 11 via intra-

\begin{tabular}{|c|c|c|c|c|c|}
\hline & $\mathrm{R}^{1}$ & $\mathrm{R}^{2}$ & $\mathrm{R}^{3}$ & $N R^{4} R^{5}$ & Yield ${ }^{\mathrm{a}}(\%)$ \\
\hline $8 a$ & $\mathrm{H}$ & $t-\mathrm{Bu}$ & $\mathrm{Ph}$ & $\mathrm{NEt}_{2}$ & 84 \\
\hline $8 b$ & $\mathrm{H}$ & $t-\mathrm{Bu}$ & $4-\mathrm{ClC}_{6} \mathrm{H}_{4}$ & $\mathrm{NEt}_{2}$ & 80 \\
\hline $8 c$ & $\mathrm{H}$ & $t-\mathrm{Bu}$ & $3-\mathrm{MeC}_{6} \mathrm{H}_{4}$ & $\mathrm{NEt}_{2}$ & 76 \\
\hline $8 d$ & $\mathrm{H}$ & $t-\mathrm{Bu}$ & $4-\mathrm{MeC}_{6} \mathrm{H}_{4}$ & $\mathrm{NEt}_{2}$ & 79 \\
\hline $8 e$ & $\mathrm{H}$ & $t-\mathrm{Bu}$ & $\mathrm{Ph}$ & morpholin-4-yl & 72 \\
\hline $8 f$ & $\mathrm{H}$ & $t-\mathrm{Bu}$ & $4-\mathrm{MeC}_{6} \mathrm{H}_{4}$ & $\mathrm{NPr}_{2}$ & 85 \\
\hline $8 g$ & $\mathrm{H}$ & $t-\mathrm{Bu}$ & 4- $\mathrm{MeC}_{6} \mathrm{H}_{4}$ & $\mathrm{NBu}_{2}$ & 69 \\
\hline $8 h$ & $\mathrm{H}$ & Cyb & $4-\mathrm{MeC}_{6} \mathrm{H}_{4}$ & $\mathrm{NEt}_{2}$ & 71 \\
\hline $8 i$ & $\mathrm{H}$ & Cyb & $\mathrm{Ph}$ & $\mathrm{NEt}_{2}$ & 86 \\
\hline 8j & $\mathrm{H}$ & $C y^{b}$ & $4-\mathrm{ClC}_{6} \mathrm{H}_{4}$ & $\mathrm{NEt}_{2}$ & 78 \\
\hline $8 k$ & $\mathrm{H}$ & Cyb & $4-\mathrm{CF}_{3} \mathrm{OC}_{6} \mathrm{H}_{4}$ & $\mathrm{NEt}_{2}$ & 80 \\
\hline 81 & $\mathrm{H}$ & $t$-Bu & $4-\mathrm{MeC}_{6} \mathrm{H}_{4}$ & morpholin-4-yl & 70 \\
\hline $8 m$ & $\mathrm{H}$ & $t-\mathrm{Bu}$ & $4-\mathrm{MeC}_{6} \mathrm{H}_{4}$ & $\mathrm{NCy}_{2}{ }^{\mathrm{b}}$ & 57 \\
\hline $8 n$ & $4-\mathrm{Cl}$ & Cyb & 4- $\mathrm{CH}_{3} \mathrm{OC}_{6} \mathrm{H}_{4}$ & $\mathrm{~N}(\mathrm{iPr})_{2}$ & 54 \\
\hline 80 & $4-\mathrm{Cl}$ & $n-\mathrm{Bu}$ & $n-\mathrm{Bu}$ & $\mathrm{N}(\mathrm{Ph}) \mathrm{Me}$ & 65 \\
\hline $8 p$ & 5-Me & $t-\mathrm{Bu}$ & Cyb & $\mathrm{N}\left(\mathrm{CH}_{2} \mathrm{Ph}\right) \mathrm{Me}$ & 74 \\
\hline $8 q$ & $4-\mathrm{Cl}$ & Cyb & $\mathrm{PhCH}_{2}$ & $\mathrm{~N}\left(\mathrm{CH}_{2} \mathrm{Ph}\right)_{2}$ & 67 \\
\hline $8 r$ & 5-Me & Cyb & $t-\mathrm{Bu}$ & $\mathrm{NEt}_{2}$ & 42 \\
\hline $8 s$ & $\mathrm{H}$ & $n-\mathrm{Bu}$ & $\mathrm{Ph}$ & $\mathrm{NPh}_{2}$ & 0 \\
\hline
\end{tabular}

alsolated yields based on the azides 4. ${ }^{b}$ Cyclohexyl. 
molecular nucleophilic substitution (Scheme 4). The results were listed in Table 3. Various secondary amines can be used in this one-pot cyclization to prepare 4H-3,1-benzothiazines 11 As indicated in Table 3, when dialkylamines including cyclic dialkylamines (Table 3, compounds $\mathbf{1 1 a}-\mathbf{k}, \mathrm{NR}^{4} \mathrm{R}^{5}=\mathrm{NEt}_{2}$, $\mathrm{NPr}_{2}, \mathrm{~N}\left(\mathrm{CH}_{2} \mathrm{Ph}\right) \mathrm{Me}, \mathrm{N}\left(\mathrm{CH}_{2} \mathrm{Ph}\right)_{2}$, piperidin-1-yl, morpholin-4$\mathrm{yl}$ and pyrrolidin-1-yl) were used, good yields (72-84\%) of the products were obtained, whereas mederate yield (48-54\%) was<smiles>[R]NC(=O)C(OC(=O)c1ccccc1)c1c[R1]ccc1N</smiles><smiles>[R]NC(=O)C(OC(=O)c1ccccc1)c1cc[R17]cc1N=P</smiles>

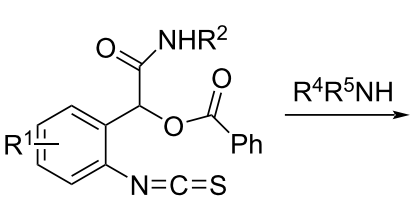

9

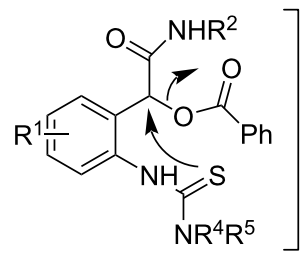
10
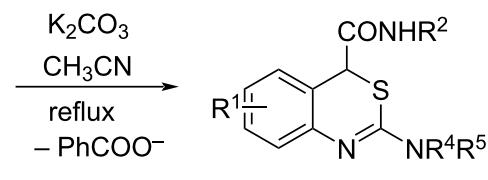

11

Scheme 4: Preparation of 4H-3,1-benzothiazines 11. obtained when the more steric dialkylamines were utilized (Table 3, compounds $\mathbf{1 1 l}$ and $\left.\mathbf{1 1 m}, \mathrm{NR}^{4} \mathrm{R}^{5}=\mathrm{N}(\mathrm{Cy})_{2}, \mathrm{~N}(\mathrm{iPr})_{2}\right)$. In cases when phenylmethylamine (compounds 11 and 11o, $\left.\mathrm{NR}^{4} \mathrm{R}^{5}=\mathrm{N}(\mathrm{Ph}) \mathrm{Me}\right)$ was used, $51-56 \%$ yields of the products were obtained, but when diphenylamine was used, no product was obtained (compound 11p, $\mathrm{NR}^{4} \mathrm{R}^{5}=\mathrm{NPh}_{2}$ ).

\section{Conclusion}

In conclusion, we have developed a new Passerini/Staudinger/ aza-Wittig/addition/nucleophilic substitution sequence for the synthesis of polysubstituted 3,4-dihydroquinazolines and 4H-3,1-benzothiazines. By this method, 3,4-dihydroquinazolines and 4H-3,1-benzothiazines were prepared in good overall yields with the advantages of mild one-pot operation conditions and easily accessible starting materials containing various common substituents.

\section{Supporting Information}

\section{Supporting Information File 1}

Experimental section and copies of NMR spectra.

[https://www.beilstein-journals.org/bjoc/content/ supplementary/1860-5397-18-32-S1.pdf]

\section{Funding}

We gratefully acknowledge financial support of this work by the National Natural Science Foundation of China (No. 21572075 ) and the 111 Project B17019.

Table 3: Yields of 4H-3,1-benzothiazines 11.

\begin{tabular}{|c|c|c|c|c|}
\hline & $\mathrm{R}^{1}$ & $\mathrm{R}^{2}$ & $N R^{4} R^{5}$ & Yielda (\%) \\
\hline $11 a$ & $\mathrm{H}$ & $t-\mathrm{Bu}$ & $\mathrm{NEt}_{2}$ & 82 \\
\hline $11 b$ & $\mathrm{H}$ & $t-\mathrm{Bu}$ & piperidin-1-yl & 83 \\
\hline $11 \mathrm{c}$ & $\mathrm{H}$ & $t-\mathrm{Bu}$ & morpholin-4-yl & 84 \\
\hline $11 d$ & $\mathrm{H}$ & $n-\mathrm{Bu}$ & morpholin-4-yl & 78 \\
\hline $11 e$ & $\mathrm{H}$ & $C y^{b}$ & pyrrolidin-1-yl & 77 \\
\hline $11 f$ & $\mathrm{H}$ & $C y^{b}$ & $\mathrm{~N}\left(\mathrm{CH}_{2} \mathrm{Ph}\right) \mathrm{Me}$ & 79 \\
\hline $11 \mathrm{~g}$ & 5-Me & Cyb & $\mathrm{NEt}_{2}$ & 72 \\
\hline $11 \mathrm{~h}$ & 5-Me & $n-\mathrm{Bu}$ & piperidin-1-yl & 81 \\
\hline $11 \mathrm{i}$ & 5-Me & Cyb & $\mathrm{N}\left(\mathrm{CH}_{2} \mathrm{Ph}\right)_{2}$ & 78 \\
\hline $11 \mathrm{j}$ & $5-\mathrm{Me}$ & $t-\mathrm{Bu}$ & $\mathrm{NPr}_{2}$ & 75 \\
\hline $11 k$ & $4-\mathrm{Cl}$ & Cy & $\mathrm{NEt}_{2}$ & 83 \\
\hline 111 & $4-\mathrm{Cl}$ & $t-\mathrm{Bu}$ & $\mathrm{NCy}_{2}{ }^{\mathrm{b}}$ & 54 \\
\hline $11 \mathrm{~m}$ & 5-Me & Cyb & $\mathrm{N}(\mathrm{iPr})_{2}$ & 48 \\
\hline $11 n$ & $\mathrm{H}$ & Cyb & $\mathrm{N}(\mathrm{Ph}) \mathrm{Me}$ & 56 \\
\hline 110 & 5-Me & Cyb & $\mathrm{N}(\mathrm{Ph}) \mathrm{Me}$ & 51 \\
\hline $11 p$ & $\mathrm{H}$ & $n-\mathrm{Bu}$ & $\mathrm{NPh}_{2}$ & 0 \\
\hline
\end{tabular}

alsolated yields based on the azides $4 .{ }^{\mathrm{b}} \mathrm{Cyclohexyl}$. 


\section{ORCID ${ }^{\circledR}$ iDs}

Ming-Wu Ding - https://orcid.org/0000-0002-3464-4774

\section{References}

1. Kim, J. H.; Jeong, H. R.; Jung, D. W.; Yoon, H. B.; Kim, S. Y.; Kim, H. J.; Lee, K.-T.; Gadotti, V. M.; Huang, J.; Zhang, F.-X.; Zamponi, G. W.; Lee, J. Y. Bioorg. Med. Chem. 2017, 25, 4656-4664. doi:10.1016/j.bmc.2017.07.010

2. Jin, K.; Sang, Y.; Han, S.; De Clercq, E.; Pannecouque, C.; Meng, G.; Chen, F. Eur. J. Med. Chem. 2019, 176, 11-20. doi:10.1016/j.ejmech.2019.05.011

3. Dukat, M.; Alix, K.; Worsham, J.; Khatri, S.; Schulte, M. K. Bioorg. Med. Chem. Lett. 2013, 23, 5945-5948. doi:10.1016/j.bmcl.2013.08.072

4. Li, W.-J.; Li, Q.; Liu, D.-L.; Ding, M.-W. J. Agric. Food Chem. 2013, 61, 1419-1426. doi:10.1021/jf305355u

5. Zhao, J.; Wang, S.; Han, S.; Kim, S. H.; Kusnetzow, A. K.; Nguyen, J.; Rico-Bautista, E.; Tan, H.; Betz, S. F.; Struthers, R. S.; Zhu, Y. Bioorg. Med. Chem. Lett. 2020, 30, 127391. doi:10.1016/j.bmcl.2020.127391

6. Jagtap, A. D.; Kondekar, N. B.; Hung, P.-Y.; Hsieh, C.-E.; Yang, C.-R.; Chen, G. S.; Chern, J.-W. Bioorg. Chem. 2020, 95, 103135. doi:10.1016/j.bioorg.2019.103135

7. Park, B.; Nam, J. H.; Kim, J. H.; Kim, H. J.; Onnis, V.; Balboni, G.; Lee, K.-T.; Park, J. H.; Catto, M.; Carotti, A.; Lee, J. Y. Bioorg. Med. Chem. Lett. 2017, 27, 1179-1185. doi:10.1016/j.bmcl.2017.01.068

8. Wiedemann, S. H.; Ellman, J. A.; Bergman, R. G. J. Org. Chem. 2006, 71, 1969-1976. doi:10.1021/jo052345b

9. Niewiadomy, A.; Matysiak, J.; Karpińska, M. M. Arch. Pharm. (Weinheim, Ger.) 2011, 344, 224-230. doi:10.1002/ardp.201000228

10. Mancini, A.; Chelini, A.; Di Capua, A.; Castelli, L.; Brogi, S.; Paolino, M.; Giuliani, G.; Cappelli, A.; Frosini, M.; Ricci, L.; Leonelli, E.; Giorgi, G.; Giordani, A.; Magistretti, J.; Anzini, M. Eur. J. Med. Chem. 2017, 126, 614-630. doi:10.1016/j.ejmech.2016.11.053

11. Matysiak, J. Bioorg. Med. Chem. 2006, 14, 2613-2619. doi:10.1016/j.bmc.2005.11.053

12. Campbell, M. V.; Iretskii, A. V.; Mosey, R. A. J. Org. Chem. 2020, 85, 11211-11225. doi:10.1021/acs.joc.0c01308

13. Kumar, R. A.; Saidulu, G.; Sridhar, B.; Liu, S. T.; Reddy, K. R. J. Org. Chem. 2013, 78, 10240-10250. doi:10.1021/j0401622r

14. Kobayashi, K.; Matsumoto, N.; Nagashima, M.; Inouchi, H. Helv. Chim. Acta 2015, 98, 184-189. doi:10.1002/hlca.201400316

15. Ren, J.; Pi, C.; Wu, Y.; Cui, X. Org. Lett. 2019, 21, 4067-4071. doi:10.1021/acs.orglett.9b01246

16. Meng, X.-H.; Yang, M.; Peng, J.-Y.; Zhao, Y.-L. Adv. Synth. Catal. 2021, 363, 244-250. doi:10.1002/adsc.202000957

17. Mishra, A.; Batra, S. Synthesis 2009, 3077-3088 doi:10.1055/s-0029-1217603

18. Gruber, N.; Díaz, J. E.; Orelli, L. R. Beilstein J. Org. Chem. 2018, 14, 2510-2519. doi:10.3762/bjoc.14.227

19. Gimbert, C.; Vallribera, A. Org. Lett. 2009, 11, 269-271. doi:10.1021/ol802346r

20. Abaev, V. T.; Tsiunchik, F. A.; Gutnov, A. V.; Butin, A. V. Tetrahedron Lett. 2006, 47, 4029-4032. doi:10.1016/j.tetlet.2006.04.010
21. Polina, S.; Putta, V. P. R. K.; Gujjarappa, R.; Singh, V.; Pujar, P. P.; Malakar, C. C. Adv. Synth. Catal. 2021, 363, 431-445. doi:10.1002/adsc.202001149

22. Sashida, H.; Kaname, M.; Minoura, M. Tetrahedron 2013, 69, 6478-6487. doi:10.1016/j.tet.2013.05.069

23. Rotstein, B. H.; Zaretsky, S.; Rai, V.; Yudin, A. K. Chem. Rev. 2014, 114, 8323-8359. doi:10.1021/cr400615v

24. Youcef, S. D.; Kerim, M. D.; Ilitki, H.; El Kaïm, L. Tetrahedron Lett. 2019, 60, 102-105. doi:10.1016/j.tetlet.2018.11.068

25. Singh, A.; Kumar, R. Chem. Commun. 2021, 57, 9708-9711. doi:10.1039/d1cc03256a

26. Jia, S.; El Kaïm, L. Eur. J. Org. Chem. 2018, 6457-6464. doi:10.1002/ejoc.201800958

27. Liu, N.; Chao, F.; Liu, M.-G.; Huang, N.-Y.; Zou, K.; Wang, L. J. Org. Chem. 2019, 84, 2366-2371. doi:10.1021/acs.joc.8b03242

28. De Moliner, F.; Bigatti, M.; Banfi, L.; Riva, R.; Basso, A. Org. Lett. 2014, 16, 2280-2283. doi:10.1021/ol500813p

29. Martinand-Lurin, E.; Dos Santos, A.; El Kaim, L.; Grimaud, L.; Retailleau, P. Chem. Commun. 2014, 50, 2214-2217. doi:10.1039/c3cc49022j

30. Pedrood, K.; Montazer, M. N.; Larijani, B.; Mahdavi, M. Synthesis 2021, 53, 2342-2366. doi:10.1055/a-1394-7511

31. Polychronidou, V.; Krupp, A.; Strohmann, C.; Antonchick, A. P. Org. Lett. 2021, 23, 6024-6029. doi:10.1021/acs.orglett.1c02099

32. Ma, X.; Zhang, X.; Awad, J. M.; Xie, G.; Qiu, W.; Muriph, R. E.; Zhang, W. Tetrahedron Lett. 2020, 61, 151392. doi:10.1016/j.tetlet.2019.151392

33. Ren, Z.-L.; Liu, J.-C.; Ding, M.-W. Synthesis 2017, 49, 745-754. doi:10.1055/s-0036-1588333

34. Wang, L.; Ren, Z.-L.; Ding, M.-W. J. Org. Chem. 2015, 80, 641-646. doi:10.1021/jo502275f

35. Wang, L.; Ren, Z.-L.; Chen, M.; Ding, M.-W. Synlett 2014, 25, 721-723. doi:10.1055/s-0033-1340596

36. Wu, J.; Zhao, L.; Yang, M.-L.; Ding, M.-W. J. Org. Chem. 2021, 86, 10755-10761. doi:10.1021/acs.joc.1c00735

37. Sun, M.; Yu, Y.-L.; Zhao, L.; Ding, M.-W. Tetrahedron 2021, 96, 132368. doi:10.1016/j.tet.2021.132368

38. Sun, M.; Yu, Y.-L.; Zhao, L.; Ding, M.-W. Tetrahedron 2021, 80, 131868. doi:10.1016/j.tet.2020.131868 


\section{License and Terms}

This is an open access article licensed under the terms of the Beilstein-Institut Open Access License Agreement (https://www.beilstein-journals.org/bjoc/terms), which is identical to the Creative Commons Attribution 4.0 International License

(https://creativecommons.org/licenses/by/4.0). The reuse of material under this license requires that the author(s), source and license are credited. Third-party material in this article could be subject to other licenses (typically indicated in the credit line), and in this case, users are required to obtain permission from the license holder to reuse the material.

The definitive version of this article is the electronic one which can be found at:

https://doi.org/10.3762/bjoc. 18.32 